\title{
High-latitude reconnection effect observed at the dayside dip equator as a precursor of a sudden impulse
}

\author{
D.-S. Han, ${ }^{1,2}$ H.-G. Yang, ${ }^{1}$ J. Liang, ${ }^{3}$ T. Iyemori, ${ }^{4}$ S. W. H. Cowley, ${ }^{5}$ and T. Araki ${ }^{1}$ \\ Received 16 August 2009; revised 9 March 2010; accepted 15 March 2010; published 14 August 2010.
}

[1] Geomagnetic sudden impulses (SI) observed at the dayside dip equator normally show a decrease and then an increase in the $H$ magnetic field component, i.e., a preliminary reverse impulse (PRI) followed by a main impulse. Using global geomagnetic field measurements, we examine an unusual SI event observed at the dayside dip equator, which shows a clear precursor $\sim 1$ min before the PRI onset. The precursor was observed simultaneously at both the dayside dip equator and in the southern polar region but was not observed at all in the northern polar region. The global ground variations after the PRI onset were, however, consistent with a conventional SI model of the magnetospheric response to a sudden enhancement of the solar wind dynamic pressure. Considering that the interplanetary magnetic field $B_{x}, B_{y}$, and $B_{z}$ components were positive with the $B_{y}$ component dominant for this event, we suggest that the SI precursor was caused by highlatitude magnetic reconnection that occurred only (or first) in the dawn quadrant of the southern hemisphere, the effect of which has for the first time been clearly identified at the dayside dip equator. This implies that electric field effects occurring in the polar ionosphere due to magnetopause reconnection may be rapidly monitored at the dayside dip equator. In addition, we argue that the quasi-simultaneous (time difference is less than $10 \mathrm{~s}$ ) appearance of the disturbance fields both in the polar region and at the dip equator confirmed in this study is extremely important for theoretically explaining the transmission of the polar electric field to the dayside dip equator, because different theories give different travel times for a disturbance field propagating from polar region to the dip equator. Our observations lend strong evidence for validity of the waveguide model.

Citation: Han, D.-S., H.-G. Yang, J. Liang, T. Iyemori, S. W. H. Cowley, and T. Araki (2010), High-latitude reconnection effect observed at the dayside dip equator as a precursor of a sudden impulse, J. Geophys. Res., 115, A08214, doi:10.1029/2009JA014787.

\section{Introduction}

[2] It is well known that a sudden enhancement of the solar wind dynamic pressure $\left(S E P_{\text {dyn }}\right)$ can cause steplike increases in the geomagnetic $H$ (northward) field component, both in the magnetosphere [e.g., Wang et al., 2007] and on the ground [e.g., Araki, 1994 and references therein]. These are normally called sudden commencements (SC) or sudden impulses (SI) depending on whether a geomagnetic storm follows or not. The local time (LT) profile of the

\footnotetext{
${ }^{1}$ SOA Key Laboratory for Polar Science, Polar Research Institute of China, Shanghai, China.

${ }^{2}$ State Key Laboratory of Space Weather, Chinese Academy of Sciences, Beijing, China.

${ }^{3}$ Department of Physics and Astronomy, University of Calgary, Calgary, Alberta, Canada.

${ }^{4}$ Data Analysis Center for Geomagnetism and Space Magnetism, Graduate School of Science, Kyoto University, Kyoto, Japan.

${ }^{5}$ Department of Physics and Astronomy, University of Leicester, Leicester, UK.

Copyright 2010 by the American Geophysical Union. 0148-0227/10/2009JA014787
}

ground level SC/SI response has been examined from low to subauroral latitudes by Russell et al. [1992, 1994a, 1994b], including major differences between southward and northward interplanetary magnetic field (IMF) conditions. On the basis of a majority of ground and satellite observations, Araki [1994] developed a physical model for SCs and suggested that there is no difference in the physical mechanisms between SIs and SCs. Araki's model can successfully explain the global ground observations of SC/SI events including anomalous [Araki et al., 1997] and negative [Araki and Nagano, 1988; Takeuchi et al., 2000] ones. Most recently, $H a n$ and $L i$ [2008] found a new feature of SC/SIs, i.e., a positive impulse always appeared at the beginning of the main impulse of the SC/SIs at the dawn time dip equator, which is also consistent with the model of Araki [1994].

[3] The content of Araki's model is summarized as follows. The source of the SC/SI in interplanetary space is a simple steplike $S E P_{\text {dyn }}$. When the $S E P_{\text {dyn }}$ impinges on the magnetosphere, a compressional (fast-mode) wave is launched and propagates antisunward into the magnetosphere. The SC/SI observed on the ground can be decomposed into 

- Canadian
+ Greenland
+ SAMNET
- TGO
- UCla
- WDC Kyoto
- Zhongshan
- BAS LPM

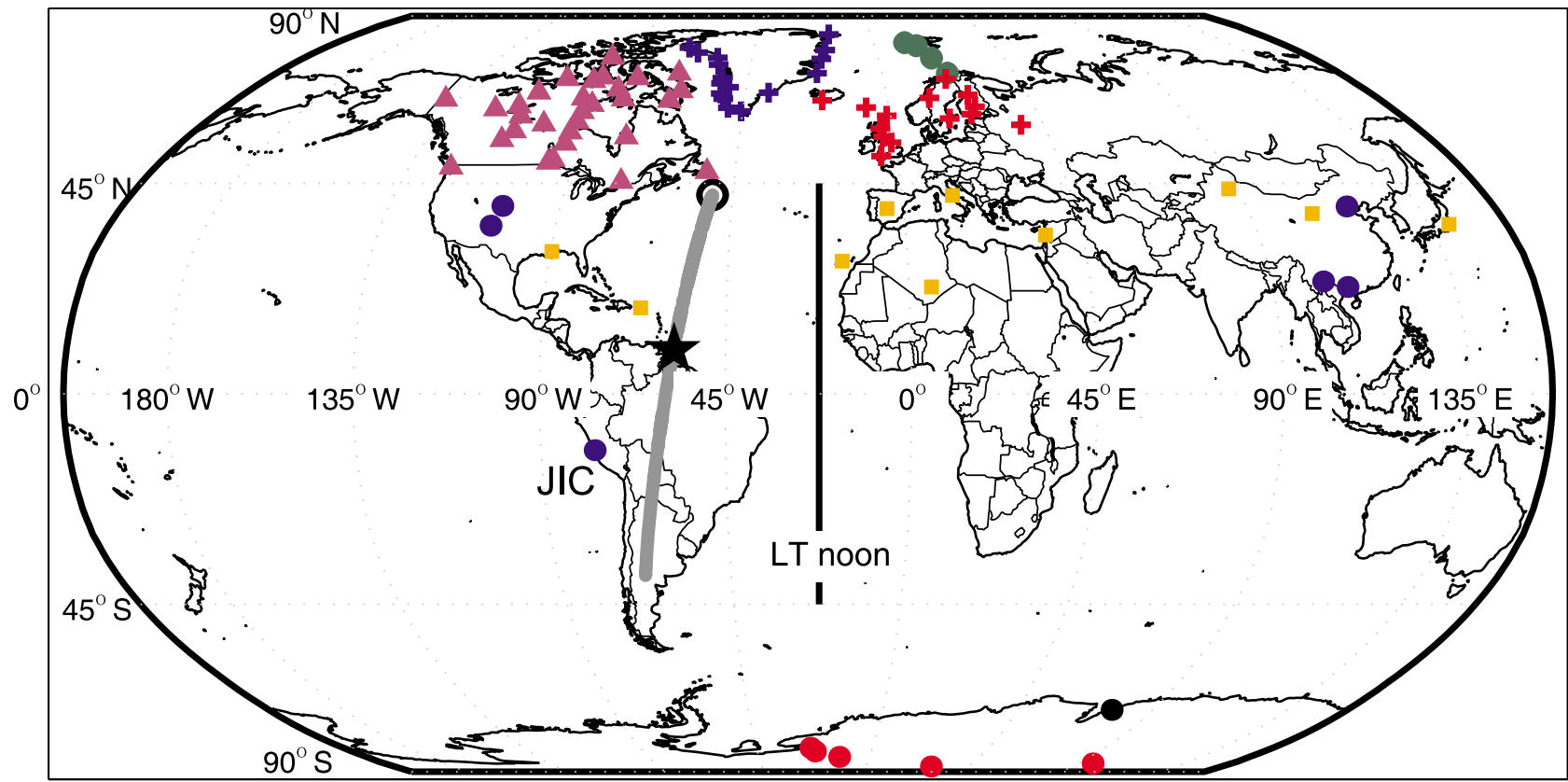

Figure 1. Location of ground stations used in this study, together with the path of the Oersted satellite. The vertical black line indicates local noon at 1330 UT on 20 March 2002. The gray curve starting with a circle shows the approximate path of the Oersted satellite. The black five-point star indicates the approximate location of the satellite at the onset of the SI.

two subfields, the DL field (disturbance dominant at low latitudes) and the DP field (disturbance field originating from the polar region). The DL field is caused by the Chapman-Ferraro currents in the dayside magnetopause and has a simple steplike structure within the magnetosphere, including on the ground. The DP field is caused by two sources. One source is the field-aligned currents (FACs) that flow into or out of the polar ionosphere accompanying the shear Alfvén waves that are converted from the compressional waves in the dayside magnetosphere. The other source is the ionospheric currents (ICs) that are generated by the electric field associated with the FACs and are twin vortex in structure in the polar region. The DP field is further decomposed into two parts corresponding to the sharp preliminary impulse $(\mathrm{PI})$ and the following main impulse (MI), called the $D P_{\mathrm{PI}}$ and $D P_{\mathrm{MI}}$ fields, respectively. The electric field imposed on the polar ionosphere associated with the FACs can be almost instantaneously transmitted to the dayside equatorial region as a zeroth-order waveguide mode [Kikuchi et al., 1978; Kikuchi and Araki, 1979], producing the DP field at the dayside dip equator with enhanced amplitudes due to the high ionospheric conductivity in that region. Hereafter we refer to this theory as the "waveguide model." The $D P_{\mathrm{PI}}$ field manifests itself as a sharp positive impulse at auroral and subauroral latitudes in the morning, but shows a negative impulse, called a preliminary reverse impulse (PRI), at auroral and subauroral latitudes in the afternoon and at the dayside dip equator [Araki, 1994, and references therein].

[4] The waveguide model is one of the key points of the model of Araki [1994]. However, this model was questioned recently by Chi et al. [2001, 2006]. Although Kikuchi and Araki [2002] disputed the work of Chi et al. [2001], the fact is that clear evidence for the simultaneous appearance of the electric field at both the dayside dip equator and the polar regions has been rarely presented up to now. In this paper, we will demonstrate that the east-west electric field can indeed be observed simultaneously at the polar and dayside dip equator regions, thus providing strong evidence for the waveguide model.

[5] Although it is well known that magnetic reconnection at the magnetopause between the IMF and the terrestrial magnetic field has a dominant effect on the Earth's magnetosphere and ionosphere as a mechanism both for plasma entry and energy input from the solar wind, reconnection effects have seldom been considered in studies of SC/SI. Boudouridis et al. [2007], however, have shown that $S E P_{\text {dyn }}$ can enhance dayside reconnection. In this paper, by examining an unusual SI event using global magnetic field observations, we will show that a $S E P_{\text {dyn }}$ may trigger high-latitude reconnection, and that the electric field in the polar ionosphere caused by the reconnection can be essentially instantaneously transmitted to the dayside dip equator. This effect has, for the first time, been clearly identified in ground magnetic field observations at the dayside dip equator as a precursor of a SI.

\section{Data Set}

[6] Ground magnetometer data obtained from various sources with different temporal resolutions are used in this study. Specifically, we employ $1 \mathrm{~s}$ resolution data from the 
Table 1. Coordinates for Some of the Ground Stations Used in This Study ${ }^{\mathrm{a}}$

\begin{tabular}{|c|c|c|c|c|c|c|c|}
\hline Data Source & Station Name & Code & Geog. Lat. ${ }^{b}$ & Geog. Long. ${ }^{\mathrm{c}}$ & Gmag. Lat. ${ }^{\mathrm{d}}$ & Gmag. Long. & $L$ Value \\
\hline \multirow[t]{4}{*}{ TGO } & Ny Ålesund & NAL & 78.92 & 11.93 & 76.26 & 110.98 & $\mathrm{~N} / \mathrm{A}$ \\
\hline & Longyarbyen & LYB & 78.20 & 15.83 & 75.31 & 111.88 & 15.80 \\
\hline & Bjørnøya & $\mathrm{BJN}$ & 74.50 & 19.00 & 71.52 & 107.76 & 10.11 \\
\hline & Sørøya & SOR & 70.54 & 22.22 & 67.40 & 106.08 & 6.88 \\
\hline \multirow[t]{10}{*}{ SAMNET } & Kilpisjärvi & KIL & 69.02 & 20.79 & 65.90 & 103.93 & 6.11 \\
\hline & Hella & HLL & 63.77 & 339.44 & 64.37 & 67.60 & 5.45 \\
\hline & Oulu & OUL & 65.10 & 25.85 & 61.65 & 105.44 & 4.52 \\
\hline & Faroes & FAR & 62.05 & 356.98 & 60.72 & 77.44 & 4.26 \\
\hline & Lerwick & LER & 60.13 & 358.82 & 58.00 & 81.04 & 3.63 \\
\hline & Nurmijärvi & NUR & 60.51 & 24.66 & 56.92 & 102.31 & 3.42 \\
\hline & Kvistaberg & KVI & 59.50 & 17.63 & 56.09 & 95.99 & 3.27 \\
\hline & Borok & BOR & 58.03 & 38.33 & 54.06 & 113.41 & 2.96 \\
\hline & Eskdalemuir & ESK & 55.32 & 356.80 & 52.69 & 77.30 & 2.77 \\
\hline & York & YOR & 53.95 & 358.95 & 50.89 & 78.51 & 2.56 \\
\hline \multirow[t]{11}{*}{ WDC Kyoto } & Ottawa & OTT & 45.40 & 284.45 & 55.83 & 355.02 & 3.44 \\
\hline & Bay St. Louis & BSL & 30.35 & 270.37 & 40.05 & 339.79 & 1.79 \\
\hline & San Juan & SJG & 18.38 & 293.88 & 28.79 & 5.90 & 1.33 \\
\hline & San Pablo-Toledo & SPT & 39.55 & 355.65 & 42.78 & 75.98 & 1.46 \\
\hline & L'Aquila & AQU & 42.38 & 13.32 & 42.42 & 94.50 & 1.55 \\
\hline & Qsaybeh & QSB & 33.87 & 35.64 & 30.23 & 113.37 & 1.29 \\
\hline & Guimar & GUI & 28.32 & 343.56 & 33.91 & 60.49 & 1.27 \\
\hline & Tamanrasset & TAM & 22.79 & 5.53 & 24.73 & 81.64 & 1.08 \\
\hline & Urumqi & WMQ & 43.80 & 87.70 & 34.11 & 162.21 & 1.65 \\
\hline & Yinchuan & YCB & 38.50 & 106.30 & 28.25 & 178.30 & 1.42 \\
\hline & Kakioka & KAK & 36.23 & 140.19 & 27.18 & 208.51 & 1.30 \\
\hline \multirow[t]{6}{*}{ UCLA } & Jicamarca & $\mathrm{JIC}$ & -12.00 & 283.10 & 0.00 & 354.20 & 1.00 \\
\hline & Boulder & BLD & 40.10 & 254.80 & 49.10 & 319.60 & 2.34 \\
\hline & Los Alamos Labs & LAL & 35.90 & 253.30 & 44.60 & 318.00 & 1.97 \\
\hline & Beijing & BJI & 40.00 & 116.20 & 28.90 & 186.10 & 1.30 \\
\hline & YongNing & YON & 22.80 & 108.50 & 11.40 & 181.10 & 1.08 \\
\hline & Tonghai & THJ & 24.00 & 102.70 & 13.00 & 174.00 & 1.05 \\
\hline \multirow[t]{5}{*}{ BAS's LPM } & M79-336 & M79 & -79.68 & 335.88 & -65.2 & 28.71 & 5.69 \\
\hline & M81-338 & M81 & -80.89 & 337.75 & -66.32 & 28.68 & 6.2 \\
\hline & M83-348 & M83 & -82.90 & 347.75 & -68.51 & 30.11 & 7.45 \\
\hline & M87-028 & M87 & -87.00 & 28.41 & -73.31 & 30.05 & 12.13 \\
\hline & M85-096 & M85 & -85.39 & 95.97 & -77.64 & 28.8 & N/A \\
\hline Zhongshan & Zhongshan & ZHS & -69.37 & 76.38 & -74.55 & 96.48 & 14.0 \\
\hline
\end{tabular}

${ }^{a}$ Canadian and Greenland station data are not given in detail.

${ }^{\mathrm{b}}$ Geographic latitude.

${ }^{\mathrm{c}}$ Geographic longitude.

${ }^{\mathrm{d}}$ Geomagnetic latitude.

${ }^{\mathrm{e}}$ Geomagnetic longitude.

UCLA Ground Magnetometer Data Center, the World Data Center for Geomagnetism in Kyoto (WDC Kyoto), the Antarctic Zhongshan (ZHS) station of China, the British Antarctic Survey Low Power Magnetometer (LPM) array, and the Sub-Auroral Magnetometer Network (SAMNET) of Lancaster University; $5 \mathrm{~s}$ data from the Canadian Magnetic Observatory System (CANMOS), Magnetometer Array for Cusp and Cleft Studies (MACCS), and Canadian Array for Real time Investigations of Magnetic Activity (CARISMA) arrays; $10 \mathrm{~s}$ data from the Tromsø Geophysical Observatories (TGO) of Tromsø University; and $20 \mathrm{~s}$ data from the Greenland magnetometers operated by the Danish Meteorological Institute. Some $1 \mathrm{~min}$ data are also provided by WDC Kyoto. Figure 1 indicates the distribution of the ground stations used in this study. Geographic and geomagnetic coordinates for some of the stations are given in Table 1.

[7] The solar wind and interplanetary magnetic field data were measured by the ACE satellite. The time resolutions of the ACE key parameters data are $\sim 64 \mathrm{~s}$ for the solar wind data and $\sim 16 \mathrm{~s}$ for the magnetic field. We also use magnetic field data from the GOES 8 satellite at 1 min resolution. Both the ACE and GOES 8 data were obtained from the
Coordinated Data Analysis Web data service (http://cdaweb. gsfc.nasa.gov/). The geostationary GOES 8 spacecraft is located at a geographic longitude of $\sim 76^{\circ} \mathrm{W}$. Vector magnetic field measurements from the Oersted spacecraft are also used in this study. Oersted is a low-altitude polar orbiting satellite, launched on 23 February 1999 with initial perigee and apogee altitudes of 638 and $849 \mathrm{~km}$, respectively. The main geomagnetic field has been subtracted from the $1 \mathrm{~s}$ measurements, and the residual data have been transformed into a local magnetic field coordinate system. The $B_{\|}$component used in this study is the field-aligned component, positive northward. The same data set has been used in the studies of Han et al. [2004, 2007]. In Figure 1, the gray curve beginning at the black circle indicates the orbit of Oersted during the interval of interest, with the black star approximately marking the location of Oersted at the SI onset.

\section{Observations}

\subsection{Introduction of the Unusual SI Event}

[8] Figure 2a shows the SI event studied in this paper as observed at Jicamarca (JIC, dip latitude $=0.0^{\circ}$ ) on the dayside on 20 March 2002 in the $H$ component. Figure $2 \mathrm{~b}$ 

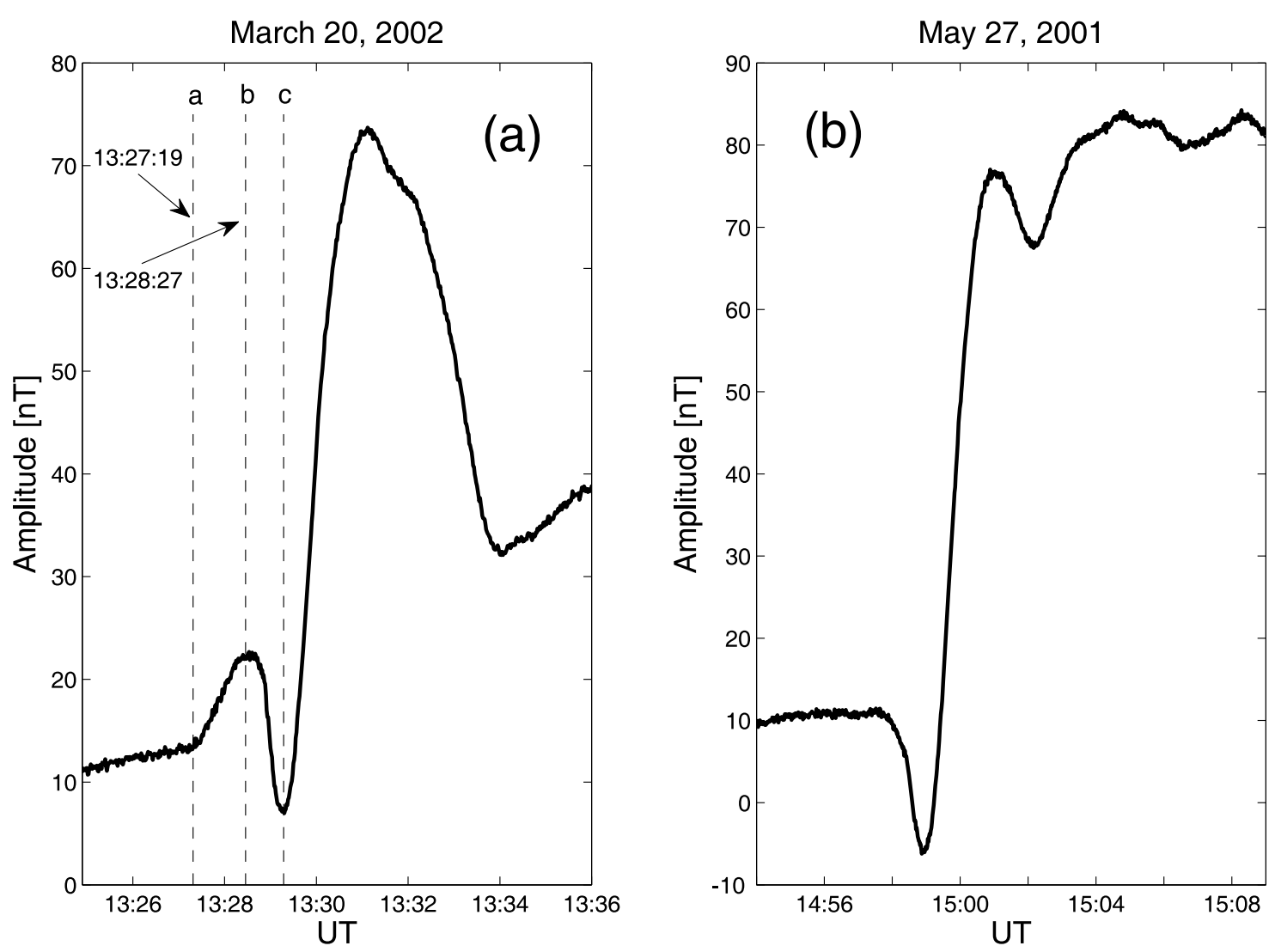

Figure 2. (a) The SI event studied in this paper observed at Jicamarca (JIC) in the $H$ component on the dayside on 20 March 2002. The vertical dashed lines a, b, and c indicate the precursor onset, the PRI onset, and the negative peak time of the PRI ( $\left.N P T_{\mathrm{PRI}}\right)$, respectively. (b) An example of a normal SC event observed at JIC in the $H$ component at nearly the same LT on 27 May 2001.

gives an example of a normal SC event observed at the same station at nearly the same LT on 27 May 2001 in the same component. The event shown in Figure $2 \mathrm{~b}$ shows a decrease, i.e., the PRI, followed by an increase, i.e., the MI, consistent with the summary of Araki [1994]. An unusual property of the SI shown in Figure 2a, however, is the clear increase observed prior to the PRI onset. In this paper we regard the field increase prior to the PRI onset as a precursor of the SI. Although there have been numerous studies on SC/SI, such a precursor of SC/SI has not attracted enough attention. This may be mainly because the precursor is often obscured by other disturbances. Even though the precursor is not a common phenomenon, that does not mean it is not important. In Figure 2a, three vertical dashed lines marked a, b, and $\mathrm{c}$ are used to indicate the precursor onset time, the PRI onset time, and the negative peak time of the PRI (NPT $\left.T_{\mathrm{PRI}}\right)$, respectively. Vertical dashed lines at the same instants as indicated here will also be given in other figures in this paper in order to make a timing comparison. The instants indicated by these dashed lines are also referred to as times $\mathrm{a}, \mathrm{b}$, and $\mathrm{c}$, respectively. We note that the time interval between time a and time $\mathrm{b}$ is $\sim 68 \mathrm{~s}$.

\subsection{Solar Wind Parameters and Observations at Geosynchronous Orbit}

[9] Figure 3 shows the upstream solar wind conditions observed by ACE in GSM coordinates, the magnetic field measured by GOES 8 at geosynchronous orbit, and the SYM- $H$ index from 1200 UT to 1600 UT on 20 March 2002. During this interval, ACE was located near (220.8, -14.9, $-14.3) R_{E}$ in $\operatorname{GSM}(x, y, z)$ coordinates. ACE detected the passage of a shock near 1305 UT. We note that the dynamic pressure has a sudden increase of $\sim 0.5 \mathrm{nPa}$. In addition, the IMF $B_{x}, B_{y}$, and $B_{z}$ components remained positive from 1200 UT to $\sim 1400 \mathrm{UT}$, with the $B_{y}$ component evidently dominant during this interval. In the magnetosphere, GOES 8 at geosynchronous orbit (on the dusk side) observed a clear SI in the $X$ and $Z$ (compressional) components. On the ground, we observe the SI in the $S Y M-H$ index. The latter index is calculated by averaging the disturbance components at six low-latitude stations distributed at different LTs, so the impulselike variation in the index indicates the global occurrence of the SI at low latitudes. Figure 3 presents a typical scenario for a sudden increase of the solar wind dynamic pressure under northward IMF conditions causing a global SI on the ground.

\subsection{Observations of the Precursor}

[10] Figure 4 shows the SI observed from subauroral latitudes to the polar region in the northern hemisphere. The left, middle, and right panels show the plots for the $H$ and $D$ components at the Canadian stations, Greenland stations, and SAMNET and TGO stations, respectively. In each panel, the lower to upper plots are basically organized by the 


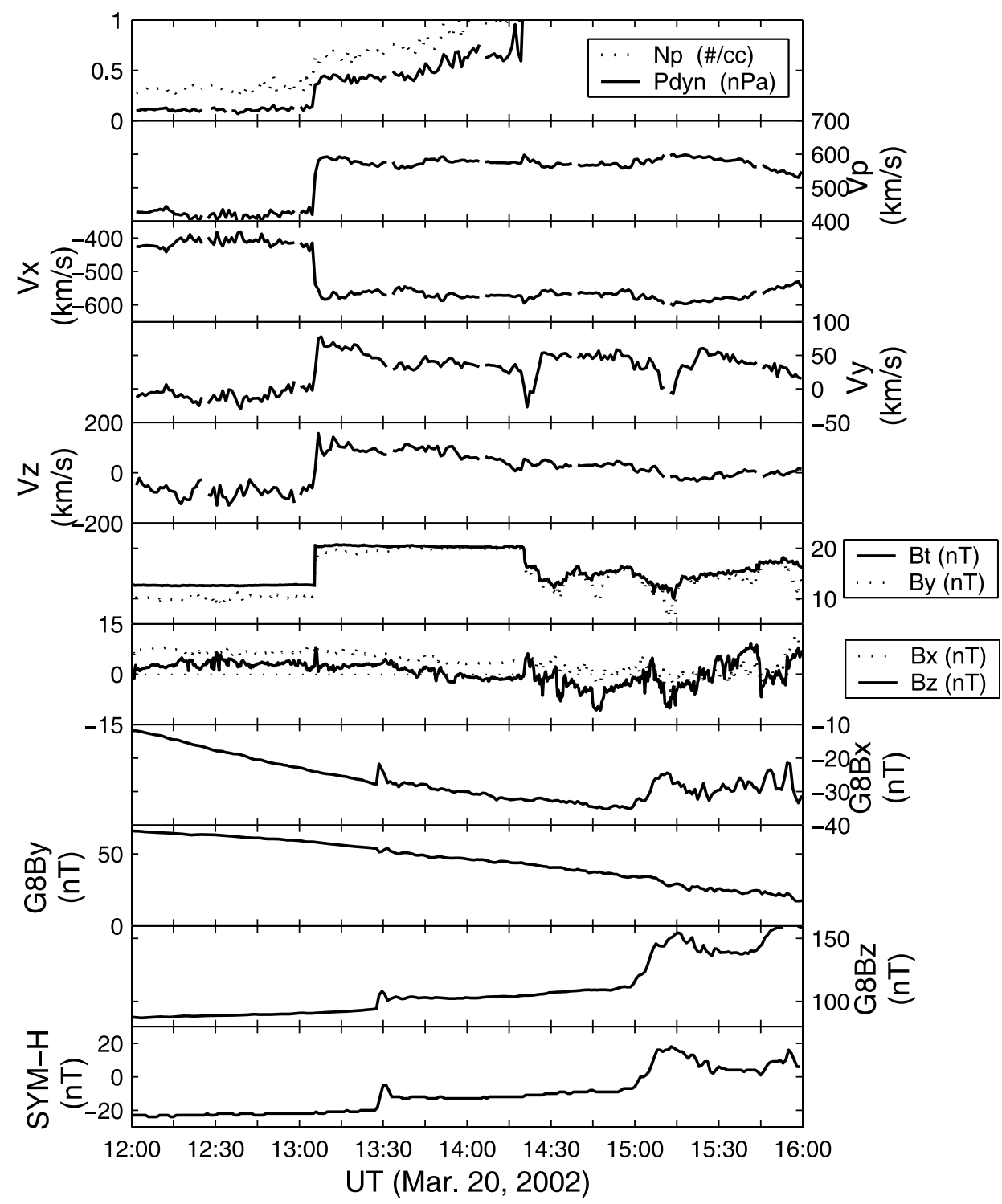

Figure 3. Solar wind and IMF parameters measured by ACE in GSM coordinates, the magnetic field observed by GOES 8 (G8B), and the SYM-H index from 1200 UT to 1600 UT on 20 March 2002.

increasing latitude of the station. From Figure 1, we notice that these stations covered almost all of the dayside LT sectors from early morning to afternoon during the SI period.

[11] Figure 5a presents the $H$ component observed at ZHS in Antarctica in the afternoon, the $H$ component at JIC at the dip equator in the morning, and the $B_{\|}$component observed above the ionosphere by Oersted at $\sim 0930$ LT. Figure $5 \mathrm{~b}$ shows the $H$ component observed at the LPM stations in Antarctica. The plots from upper to lower are organized by the increase in $L$ value of the station. It should be noted that all the LPM stations were located in the prenoon sector during the SI period, as shown in Figure 5c.

[12] In this subsection, we focus on examining the appearance of the precursor at the dayside dip equator and in the polar regions as presented in Figures 4 and 5. Figure 4 shows that when the SI onset was observed at JIC at time a, neither the $1 \mathrm{~s}$ observations at the SAMNET stations nor the $5 \mathrm{~s}, 10 \mathrm{~s}$, and $20 \mathrm{~s}$ observations at other stations showed any disturbance in either the $H$ or $D$ components (all these stations are in the northern hemisphere). However, Figure 5 shows that all of the southern antarctic LPM stations, as well as ZHS, simultaneously observed a clear decrease in the $H$ component at time a. Thanks to the quiet magnetic activity prior to the SI, Figures 4 and 5 clearly confirm that the precursive disturbance was observed simultaneously at the dip equator and in Antarctica but was not observed in the polar region of the northern hemisphere. Such asymmetry between the two hemispheres, as far as we know, has never been noticed in SIs, such that its generation mechanism is worthy of investigation. Intuitively, one might think that it could be caused by a propagation effect, i.e., that the disturbance field generated on the magnetopause travelled to the southern hemisphere earlier than to the northern hemisphere. However, we will demonstrate that this asymmetry was caused by a non-conjugate process that occurred only, or first, in the southern hemisphere. 

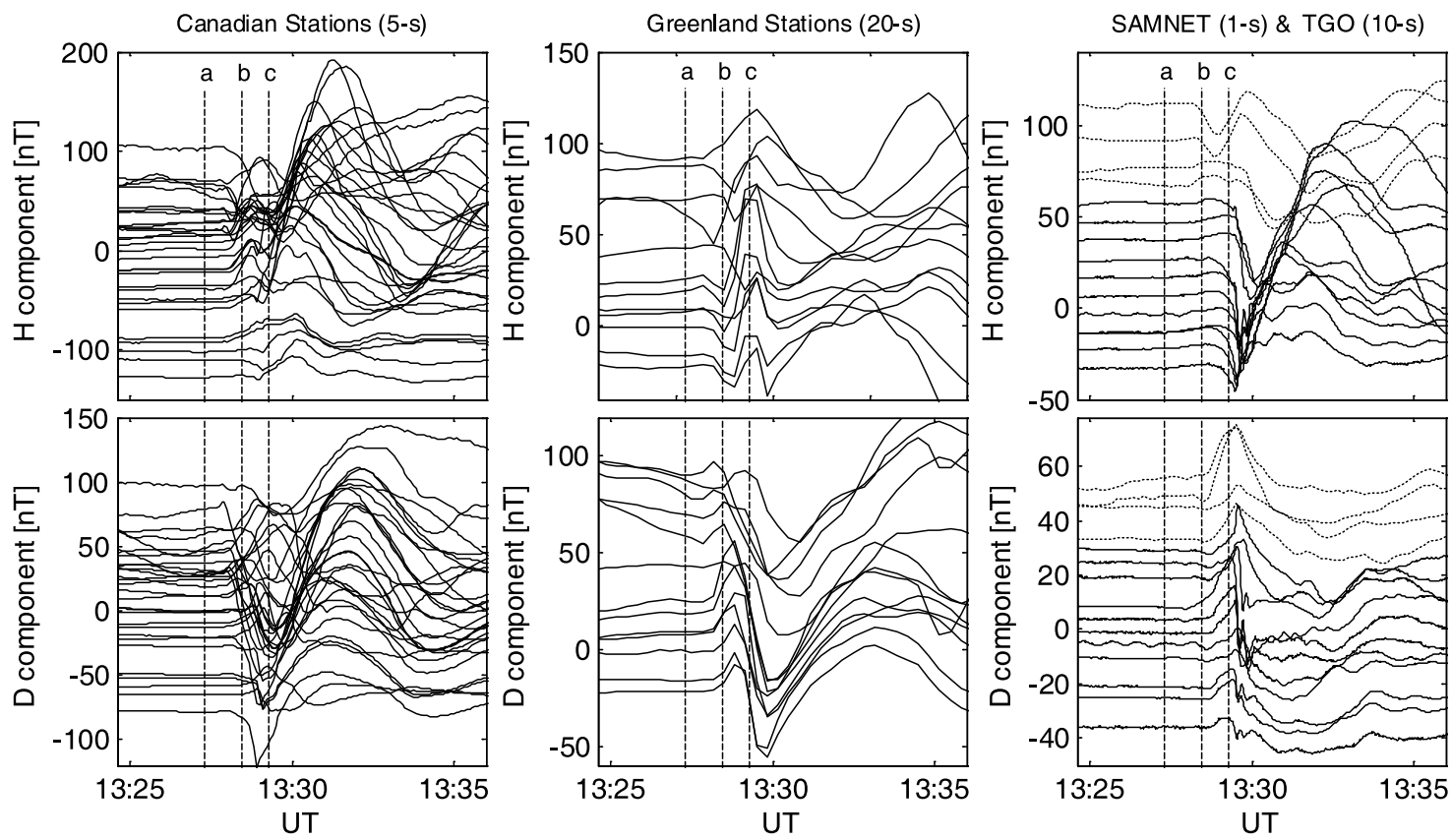

Figure 4. Observations of the SI from subauroral latitudes to the polar region in the northern hemisphere. The left, middle, and right panels show plots for the $H$ and $D$ components at the Canadian stations, Greenland stations, and Sub-Auroral Magnetometer Network (SAMNET) and Tromsø Geophysical Observatories (TGO) stations, respectively. In each panel, the lower to upper plots are basically organized by the increase in latitude of the station.

\subsection{Global Observations After the PRI Onset}

[13] In this subsection, we focus on presenting two observational facts about the global observations after the PRI onset. One is the global appearance of the PRI signal close to time $\mathrm{b}$ and another is the agreement of the global observations with the conventional SC/SI model of Araki [1994].

[14] Figure 4 (right) indicates that when the initial SI signal was observed at the SAMNET and TGO stations at

(a)

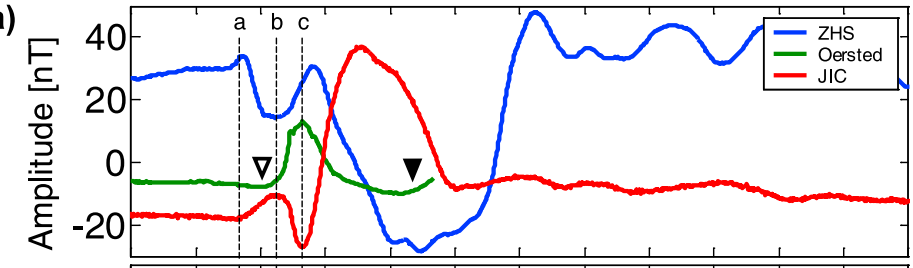

(b)

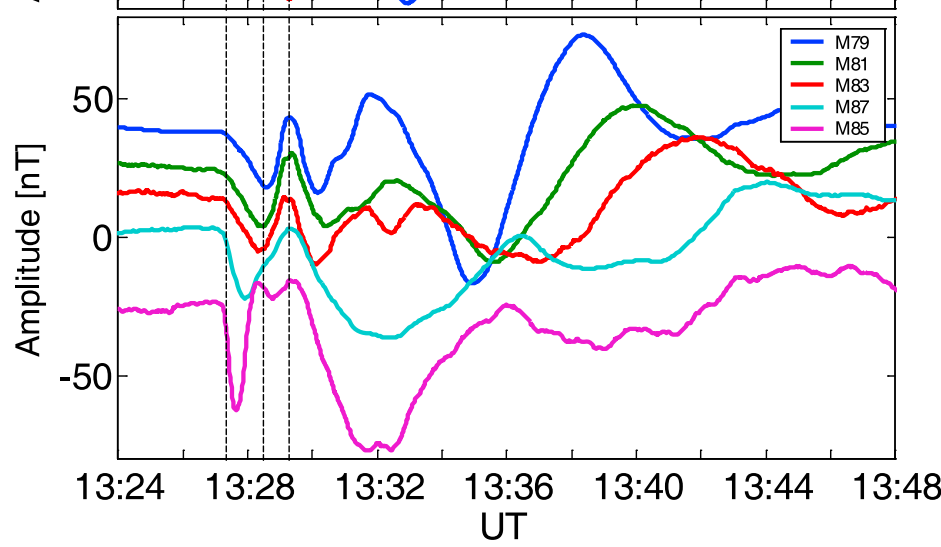

(c) Location of the Antarctic stations in geomagnetic coordinates

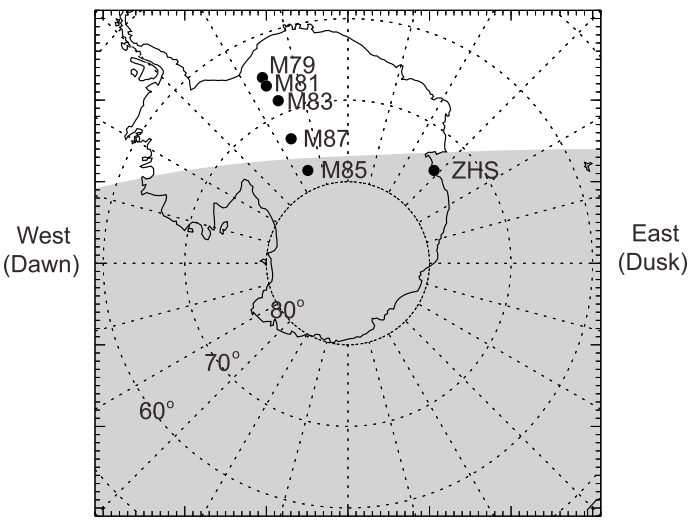

Figure 5. (a) The $H$ component observed at Zhongshan (ZHS) in Antarctica in the afternoon, the $H$ component at JIC at the dip equator in the morning, and the $B_{\|}$component observed above the ionosphere by Oersted at $\sim 0930$ LT. (b) The $H$ component observed at the LPM stations in Antarctica. The plots from upper to lower are organized by increasing $L$ value of the station. (c) Relative location of the stations in Antarctica in geomagnetic coordinates. Note that the LPM stations were located in the prenoon sector during the event. 


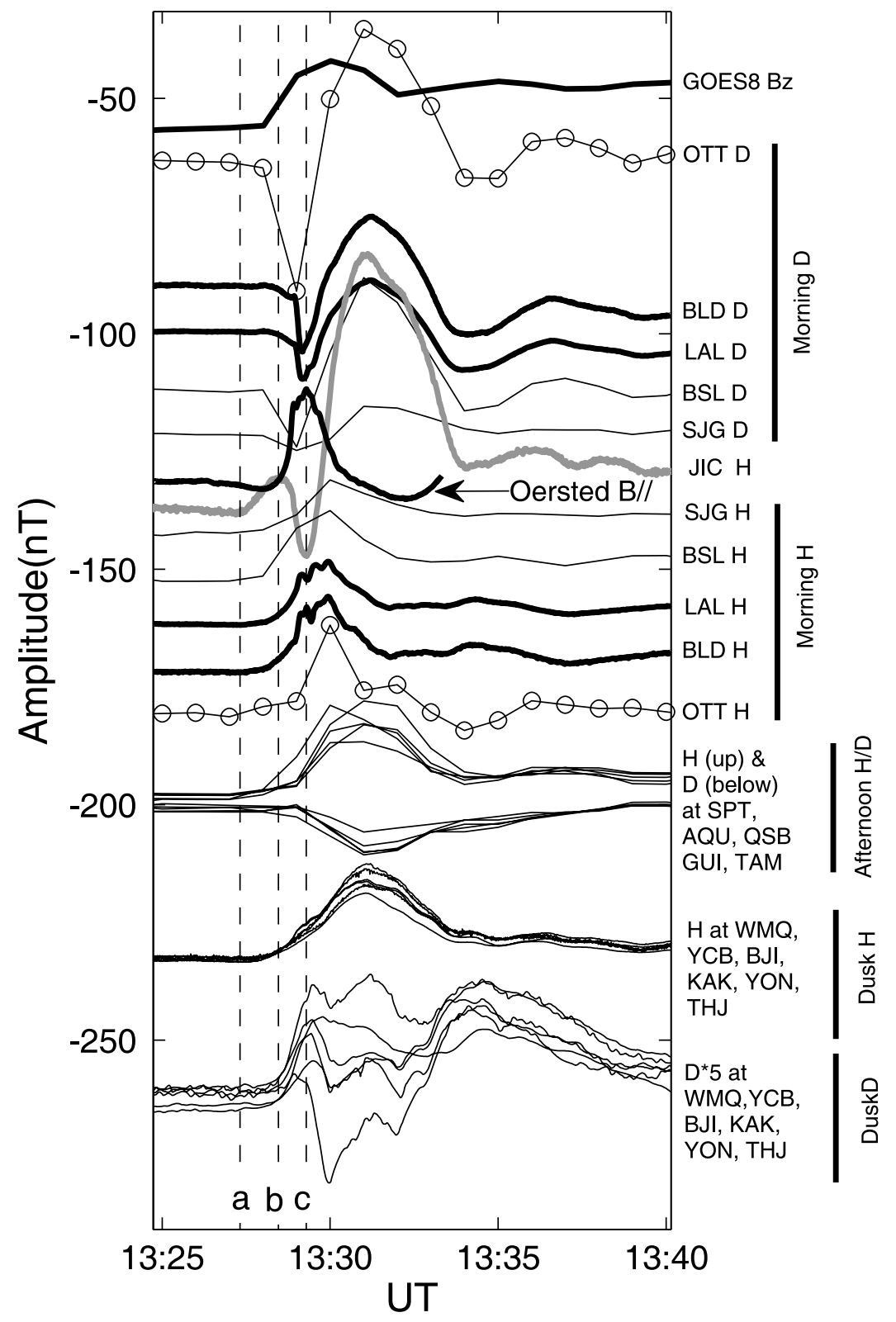

Figure 6. The SI observed at low latitudes in the morning, early afternoon, and dusk/night sector. From top to bottom we show the $D$ components in the morning sector from higher to lower latitudes, the $H$ component at JIC, the $H$ component in the morning sector from lower to higher latitudes, the $H$ and $D$ components in the early afternoon sector, the $H$ component in the dusk sector, and the $D$ component (with amplitude increased by a factor of 5) in the dusk/night sector, respectively. The compressional magnetic field component $\left(B_{z}\right)$ observed at geosynchronous orbit by GOES 8 is given at the top of the plot, shifted forward in time by $30 \mathrm{~s}$.

time $b$, a corresponding response, i.e., the PRI onset, was observed at the dayside dip equator at JIC. At the same time, Figure $5 \mathrm{~b}$ shows that a corresponding response was observed at the LPM stations in Antarctica at time $b$ too. Furthermore, Figure 6 presents the SI observed at middle and low latitudes in the morning, afternoon, and dusk/night sectors. Figure 6 shows that the precursor onset was not observed at the low- and mid-latitude stations in the northern hemisphere at time a and that a global response was only observed from time $b$. These observational facts strongly indicate that a global disturbance, which may be caused by a common source, was simultaneously observed at time b. Previously, we showed that when the precursor was observed in the southern polar region and at the dayside dip equator at time a, the northern polar region did not observe any disturbance. By integration of these observational results, we suggest that the variations observed at time $\mathrm{b}$ in the northern polar region (the SI onset), at the dip equator (the PRI onset), and in the southern polar region (a corresponding disturbance) are caused by a common 
source, while the precursive disturbances observed from time $a$ to time $b$ in the southern polar region and at the dip equator were caused by a different source. Thus, the global observations for this event are likely caused by two independent processes. One occurred only in the southern hemisphere, leading to the ground responses observed from time a, while the other caused the global ground response from time $b$.

[15] For normal SC/SI events, Araki's model [Araki, 1994] can well explain the global ground response to a $S E P_{\text {dyn. }}$. The main physical processes involved in this model have been summarized in section 1. Here we suggest that the global variations observed after time $b$ for this event reflect the normal scenario for the ground response to a $S E P_{\mathrm{dyn}}$, because they are clearly consistent with the model predictions of Araki [1994]. According to Araki's model, the variations from times $\mathrm{b}$ to $\mathrm{c}$ and after time $\mathrm{c}$ should correspond to the $D P_{\mathrm{PI}}$ and $D P_{\mathrm{MI}}$ fields, respectively. Araki [1994] suggests that both the $D P_{\mathrm{PI}}$ and the $D P_{\mathrm{MI}}$ fields can be explained by an equivalent ionospheric current (IC) system in the pattern given in Figure 10 of Araki [1994]. Araki also suggests that the equivalent ICs for the $D P_{\mathrm{PI}}$ and $D P_{\mathrm{MI}}$ fields have the same pattern but with opposite polarity. Figures $7 \mathrm{a}$ (top) and $7 \mathrm{~b}$ (top) show the equivalent ICs estimated from the dayside observations for the period from time b to time c (i.e., the $D P_{\mathrm{PI}}$ field of the SI) and after time c (i.e., the $D P_{\mathrm{MI}}$ field of the $\mathrm{SI}$ ), respectively. The patterns of the equivalent ICs depicted in Araki's model for the $D P_{\mathrm{PI}}$ and $D P_{\mathrm{MI}}$ fields are correspondingly shown at the bottom of each panel. Figure 7 indicates that the global variations observed after time $\mathrm{b}$ are basically consistent with the model of Araki [1994]. In fact, this consistency is also perfectly reflected in the low- and mid-latitude observations shown in Figure 6. For example, from time $b$ to time $c$, the afternoon vortex should be clockwise as shown in Figure 7 , and it should produce decreases both in the LAL/BLD $D$ component and in the JIC $H$ component. This explains why the variations of these components are highly coherent as shown in Figure 6. At the same time, the IC vortex should also produce increases and decreases in the $H$ component in the morning (e.g., at LAL and BLD) and afternoon (e.g., at SPT, AQU, QSB, GUI, and TAM), respectively. Superposing these disturbances on the increasing DL field can well explain why the increase in the $H$ component observed in the morning was steeper than that observed in the afternoon during this time period. We therefore conclude that the global ground variations observed after time $\mathrm{b}$ were caused by the same process as that generating normal SC/SI events as described in Araki [1994].

[16] Another point which should be noted is that although Figure 4 shows that the SI onsets observed at the Canadian and Greenland stations occurred close to time b, the stations at higher latitude tended to observe the SI onset earlier. For example, the initial SI disturbance observed at the highlatitude Canadian stations occurred prior to time b, clearly earlier than that observed at the low-latitude Canadian stations. This time lag is most likely caused by the fact that the Alfvén speed in the outer magnetosphere is higher than that in the inner magnetosphere, so that the disturbance signals generated by compression of the magnetopause can arrive at the higher-latitude stations a little earlier. This effect has been discussed by Chi et al. [2006] and does not conflict with the Araki [1994] model.

\section{Discussion}

[17] In the previous section, we showed that the global observations after the PRI onset reflect the normal global ground responses to a $S E P_{\text {dyn }}$. This has been extensively examined in previous studies of SC/SIs as summarized in Araki [1994] and will not be discussed here. In this section, we will mainly focus on the following topics, first analyzing the electric fields that were generated both in the southern polar region and at the dayside dip equator during the SI period, and second discussing the possible processes for generating the precursor of the SI.

\subsection{Electric Fields Inferred From Magnetic Observations at the Dayside Dip Equator}

[18] In Figure 5a, dashed lines $\mathrm{b}$ and $\mathrm{c}$ indicate that the PRI observed in the JIC $H$ component on the ground is in antiphase to that observed in the Oersted $B_{\|}$component above the ionosphere. The open and solid inverted triangles over the plot of the Oersted $B_{\|}$component indicate the instants when the satellite was at $15.0^{\circ}$ and $0.0^{\circ}$ in magnetic latitude, respectively. The star in Figure 1 approximately indicates the location of Oersted when the SI onset was observed. We note that the satellite was nearly overhead of JIC during the period of the SI. Although Han et al. [2007] has made comparative studies of SC/SI perturbations observed by low-altitude satellites and from ground-based magnetic field measurements, this is the first time that a PRI disturbance of an SI has been investigated in detail by simultaneous measurements from above and below the dayside ionosphere.

[19] By comparing the observations presented in Figure 6, we note that the SI observed in the compressional $\left(B_{z}\right)$ component at geosynchronous orbit by GOES 8 clearly differs from that observed just above the ionosphere by Oersted. The gradient of the Oersted $B_{\|}$component $\left(\Delta B_{\|} / \Delta t\right)$ is greater than that of the GOES $8 B_{z}$ component $\left(\Delta B_{z} / \Delta t\right)$. In addition, the duration of the impulse (calculated from onset of the increase to end of the decrease) observed in the GOES 8 component is longer than that observed in the Oersted $B_{\|}$component. We believe that these differences reflect the effects of the ICs, otherwise the SI would have similar waveforms in the Oersted $B_{\|}$and GOES $8 B_{z}$ components. As discussed in section 1, Araki [1994] suggested that the observation of SCs on the ground can be decomposed into two disturbance fields, i.e., the DL field caused by the magnetopause currents and the DP field produced by ICs and FACs. Because the altitude of Oersted is just above the ionosphere, the SI observed by Oersted should certainly consist of the effects of both the DL and the DP fields (here we only consider the DP fields that were caused by the ICs, because the effect of FACs is small by comparison at the dip equator). Dashed lines $\mathrm{b}$ and $\mathrm{c}$ in Figure $5 \mathrm{a}$ demonstrate that the variations in the Oersted $B_{\|}$and JIC $H$ components are in antiphase after time $b$. These observations naturally lead us to consider that a westward IC was induced from time $b$ at the dip equator. This westward IC reached its maximum magnitude at time c, i.e., the $N P T_{\mathrm{PRI}}$, and then a net eastward IC appeared. Thus, when the westward IC produced 
(a) Equivalent IC for the DPPI field: from time ' $b$ ' to ' $c$ '
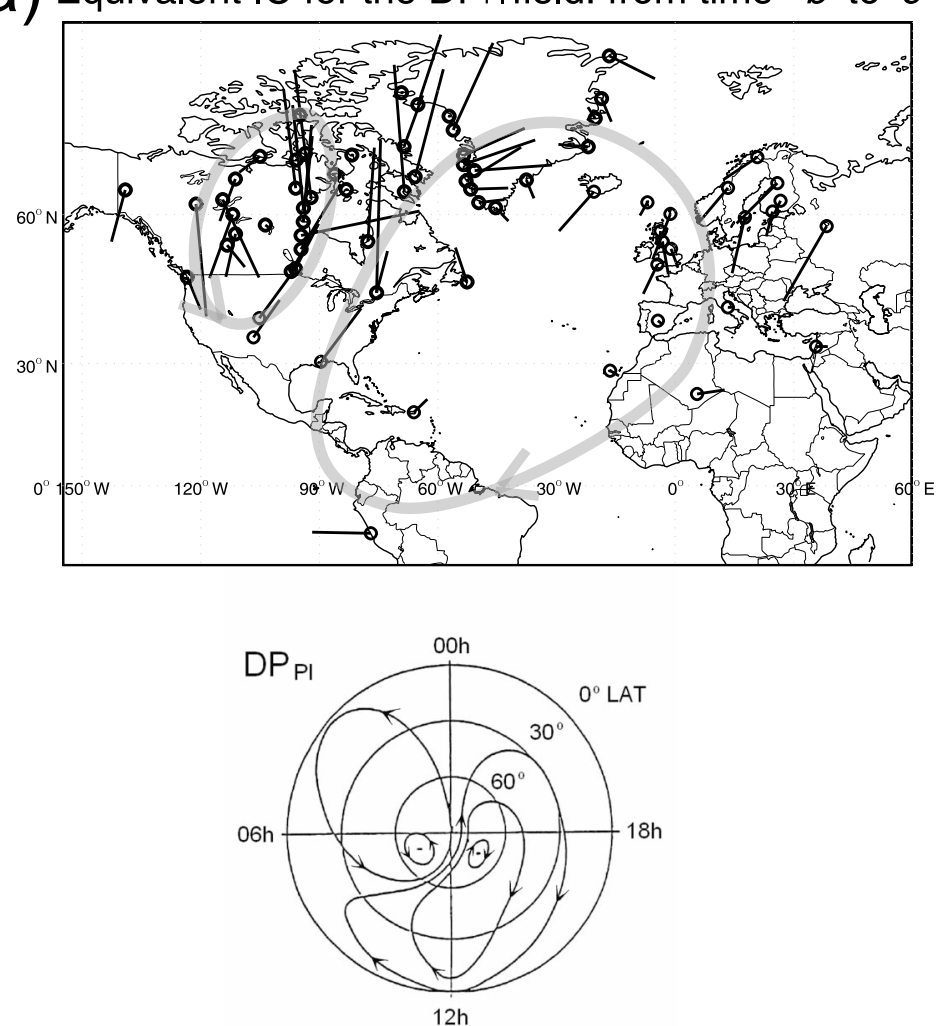

(b) Equivalent IC for the DPMI field: after time ' $c$ '
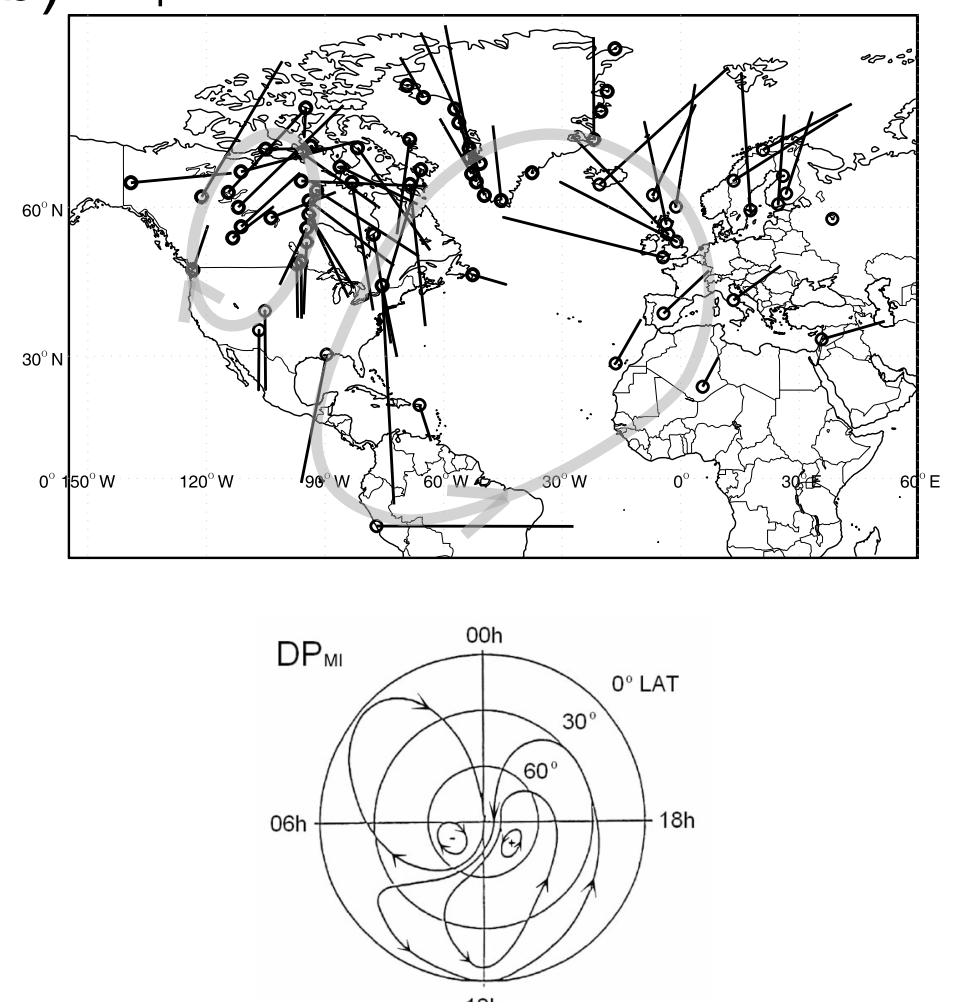

$12 \mathrm{~h}$

Figure 7. (a) and (b) Equivalent ionospheric currents estimated from the dayside observations for the period from time b to c (i.e., the $D P_{\mathrm{PI}}$ field of the $\mathrm{SI}$ ) and after time c (i.e., the $D P_{\mathrm{MI}}$ field of the $\mathrm{SI}$ ), respectively. The patterns of the equivalent ionospheric currents depicted in Araki's model for the $D P_{\mathrm{PI}}$ and $D P_{\mathrm{MI}}$ fields are correspondingly given in the bottom panels. 
the decrease in the JIC $H$ component from time b, it also produced an increase in the $B_{\|}$component above the ionosphere. This increase in the $B_{\|}$component superposed on the increasing DL field led to the increase of the Oersted $B_{\|}$ component becoming much steeper (as shown in Figure 5a). After time c, the eastward IC produced a decrease in the $B_{\|}$ component above the ionosphere. The magnitude of this decrease must be greater than that of the increase of the DL field during this time period, so that their superposition led to the Oersted $B_{\|}$component decreasing quickly from time c. This also explains why the duration of the SI observed by Oersted is less than that observed by GOES 8 (it should be noted that the DL fields caused by the magnetopause currents should have the same waveform at GOES 8, Oersted, and JIC, being only different in amplitude). In addition, this eastward IC also produced an increase in the ground $H$ component, which, superposed on the increasing DL field, made the amplitude of the JIC $H$ component become much greater than that observed at other low-latitude stations, due to the high ionospheric conductivity at the dip equator. We therefore argue that the simultaneous observations from above and below the ionosphere have demonstrated that a westward IC was generated at the dayside dip equator from the PRI onset, and an eastward IC did appear after the $N P T_{\mathrm{PRI}}$. From only the ground magnetic observations, we can only estimate an equivalent IC. That is, we cannot discriminate if the current is actually flowing in the ionosphere or if it is just an equivalent effect of currents in the space. Here we confirmed the existence of these ICs by simultaneous observations from above and below the ionosphere, so we argue that they were not equivalent ones, but actually flowed in the ionosphere. Confirmation of such fine structure of the ICs is one of the merits of simultaneous observations from both above and below the ionosphere.

[20] For the precursive disturbance observed in the $H$ component at JIC from time a to time $b$, it is reasonable to assume that it was caused by an eastward IC in the dip equator. Ideally, the existence of this eastward IC should also be identified by an antiphase variation between Oersted and JIC. However, Figure 5a does not show a clear decrease in the Oersted $B_{\|}$component corresponding to the increase observed at JIC. This could be due to two reasons. First, Figure 5a shows that the amplitude of the increase from time a to time $\mathrm{b}$ in the JIC $H$ component is rather small, which implies that the magnitude of the eastward IC in the dip equator ionosphere should be small too. Considering that the altitude of the satellite is higher than $600 \mathrm{~km}$, if we assume that the altitude of the IC is at $\sim 110 \mathrm{~km}$, the amplitude of the disturbance field generated by the IC at Oersted will be much smaller than that at JIC. Second, the open inverted triangle in Figure 5a indicates that Oersted was located near $\sim 15^{\circ}$ in magnetic latitude during the interval of the precursor. If the precursor observed at JIC was caused by an IC that was in-linelike and localized in the dip equator, the magnetic effect will be small at Oersted latitudes. These could be the reasons why the Oersted satellite did not observe a clear decrease in the $B_{\|}$component from time a to $\mathrm{b}$, but it does not affect the validity of our assumption that the precursor observed at JIC was caused by an eastward IC.

[21] In summary, on the basis of these observations, we have inferred that eastward, westward, and then eastward electric fields were generated in the dip equator ionosphere following times $\mathrm{a}, \mathrm{b}$, and $\mathrm{c}$, respectively.

\subsection{Electric Field in the Southern Polar Region}

[22] Figure 5b shows that the precursor onset observed at the LPM stations in Antarctica in the prenoon sector was almost simultaneous with that observed at JIC at the dayside dip equator, which strongly implies that they could be caused by a common source. It should be noted that the mechanism for quasi-instantaneous transmission of the polar electric field to the dayside dip equator has previously been proposed by Kikuchi et al. [1978] in terms of a waveguide model. Therefore, here we assume that the precursive disturbances observed at JIC and at the LPM stations were both caused by an eastward electric field in the ionosphere, which was generated in the southern polar region and then quasiinstantaneously transmitted to the dayside dip equator. Now we first examine if such an eastward electric field in the southern polar region is consistent with our observations or not, and then we consider how it is generated.

[23] We know that a pair of FACs that flow into the polar ionosphere in the morning and flow out of the ionosphere in the afternoon can lead to an eastward (dawn-to-dusk) electric field in the polar ionosphere. Associated with the eastward electric field in the southern polar ionosphere, a counterclockwise Hall current vortex will be generated in the morning sector (seen from above the South Pole as schematically depicted later in Figure 10), and the current vortex center would correspond with the footprint of the downward FAC. Note that the LPM stations were located in the morning sector in the southern polar region and observed decreases in the $H$ component after the precursor onset. Therefore, only if the current vortex center is located poleward of the LPM stations, the Hall currents will generate decreases in the $H$ components at these stations and thus lead to the variations as shown in Figure 5b. The location of the current vortex center can be inferred from two observational facts we will now discuss. First, as shown in Figure 5b, soon after time a, all of the LPM stations without exception observed decreases in the $H$ component, which implies that the latitude of the current vortex center cannot lie within the range of latitudes of these stations at this time. Noting also that the amplitude of the decrease in the $H$ component decreases from higher to lower latitude, we infer that the current vortex center must be poleward of these stations rather than equatorward. Second, between times a and b, observations at stations M85 and M87 at higher latitudes show a decrease and then an increase, which differs from the monotonic decreases observed at M79, M81, and M83 at lower latitudes. In addition, the negative peak of M85 leads that of M87. From these observations, we infer not only that the current vortex center was located poleward of M85 at time a, but also that it moved gradually equatorward over M85 and M87 during the interval from time a to time $b$. Taking this movement of the current vortex center into account, we can successfully interpret the observations at M85 and M87 during the precursive disturbance shown in Figure $5 \mathrm{~b}$, because the Hall current will produce a decrease (increase) in the $H$ component at a ground station when the current-vortex center is poleward (equatorward) of it. Thus, we can say that the precursive disturbance observed at the LPM stations was indeed associated with an eastward 
electric field. Namely, it is in the same direction as the electric field required to generate the precursive disturbance observed at JIC at the dayside dip equator.

\subsection{Generation of the Polar Electric Field for the Precursor of the SI}

[24] Up to now, although we showed that an eastward electric field in the southern polar region is consistent with our observations for the precursor, we still did not discuss how the electric field was generated. In previous subsections, we presented that the SI precursor was observed simultaneously at the dayside dip equator and in the southern polar region but was not observed at all in the northern polar region. We therefore suggested that the precursor was caused by a nonconjugate process that occurred only in the southern hemisphere but not in the northern hemisphere. Considering that the IMF $B_{x}, B_{y}$, and $B_{z}$ components for this event were positive and that the $B_{y}$ component was dominant (as shown in Figure 3), we suppose the nonconjugate process could be high-latitude magnetic reconnection, which occur only, or first, in the southern hemisphere. It is well known that magnetic reconnection between the IMF with the geomagnetic field has a dominant effect on ionospheric electric fields and currents and that the reconnection properties significantly depend on both the strength and direction of all three components of the IMF [e.g., Cowley, 1983]. When the IMF $B_{z}$ and $B_{y}$ components are positive, high-latitude lobe reconnection is favored to occur in the dawn quadrant of the southern hemisphere and in the dusk quadrant of the northern hemisphere [e.g., Watanabe and Sofko, 2009]. In addition, when the IMF $B_{x}$ component is positive, high-latitude reconnection may occur in only one hemisphere [Fuselier et al., 1995, 1997; Lavraud et al., 2005; Lockwood and Moen, 1999]. High-latitude reconnection occurring only in the dawn quadrant of the southern hemisphere is just what we expect for interpreting our observations. Our interpretation is likely also valid if high-latitude reconnection occurs in both hemispheres as long as there is a sufficient delay between southern and northern hemisphere reconnection initiation. For northward IMF, reconnection may occur in both hemispheres [Lavraud et al., 2006] but with a delay that depends on both the IMF $B_{x}$ and the dipole tilt. Lavraud et al. [2005] show that the dipole tilt determines which hemisphere reconnects first for large dipole tilts, but for small dipole tilts the IMF $B_{x}$ may be more important. For this event, the dipole tilt is $\sim 6.5$, which should favor northern hemisphere reconnecting first [Lavraud et al., 2005]. But because this is a low tilt value, IMF $B_{x}$ may dominate and thus reconnection may occur first in the southern hemisphere.

[25] Although we cannot provide direct observational evidence for the occurrence of high-latitude reconnection in the southern hemisphere, Twitty et al. [2004] found that for positive IMF $B_{z}$, the signatures of high-latitude reconnection in the cusp were observed $\sim 90 \%$ of the time. In addition, results of a real event 3-D MHD simulation using the Block-Adaptive-Tree-Solarwind-Roe-Upwind-Scheme (BATSRUS) code provided by the Community Coordinated Modeling Center (CCMC) also strongly support our suggestion. We took the solar wind parameters as shown in Figure 3 as the model inputs, adding an appropriate time shift to ensure that the time of the interaction between the $S E P_{\text {dyn }}$ and the magnetosphere matches our observations. Figure 8 presents the simulation results of the magnetic field topology viewed at $X=15 R_{E}$ in the Y-Z plane in GSM coordinates. Figure 9 gives the simulation results of the ionospheric electric potential in both hemispheres. In Figures 8 and 9, we set the moment that the shock arrives at $X=15 R_{E}$ (identified by a sudden increase of the IMF $B_{y}$ component) as Time $=0 \mathrm{~min}$ as a reference, and show plots from Time $=-1 \mathrm{~min}$ to Time $=4 \mathrm{~min}$, respectively. Figure 8 shows that at Time $=2 \mathrm{~min}$, i.e., $2 \mathrm{~min}$ after the shock arrived at $X=15 R_{E}$, high-latitude reconnection occurred in the dawn quadrant of the southern hemisphere. Occurrence of the reconnection is consistent with our expectation. Furthermore, Figure 9 shows that the ionospheric electric potential in the southern hemisphere suddenly increased at Time $=2 \mathrm{~min}$ and that the high potential center moved equatorward from Time $=2 \mathrm{~min}$ to Time $=4 \mathrm{~min}$. It should be noted that this equatorward motion of the high potential center is perfectly reflected in our observations shown in Figure 5b, which have been discussed in the previous subsection.

[26] In summary, the processes for generation of the SI as discussed above can be schematically represented in Figure 10. Step 1 in Figure 10 illustrates the process for generation of the precursor from time a to time b. Step 1 (left) indicates that the shock first triggered high-latitude reconnection (or caused increase of reconnection rate) in the southern hemisphere. Step 1 (middle) illustrates that the reconnection led to a dawn-to-dusk (eastward) electric field in the southern polar region. With the reconnected field line draped from poleward cusp toward equator, the resultant high potential center in the ionosphere also moved equatorward (as discussed in previous subsection). Step 1 (right) shows the ground response in the southern polar region observed at the LPM stations, as well as at the dayside dip equator observed at JIC, during this time period. In Step 2 and Step 3 of Figure 10, the global magnetospheric responses to the $S E P_{\text {dyn }}$ are well consistent with the model of Araki [1994], which will not be explained in detail here.

[27] In addition to the discussions presented above, we stress that our observations strongly imply that the shock indeed triggered the high-latitude reconnection or dramatically increased the reconnection rate. Figure 3 shows that the IMF $B_{x}, B_{y}$, and $B_{z}$ components had been continuously positive for more than $1 \mathrm{~h}$ before the shock. Although these IMF conditions favor high-latitude reconnection occurring

Figure 8. Real event simulation results of the Block-Adaptive-Tree-Solarwind-Roe-Upwind-Scheme (BATSRUS) model for the magnetic field topology viewed at $X=15 R_{E}$ in the Y-Z cut plane in GSM coordinates. Plots of color contours, vectors, and vector flow lines of the IMF are shown here. The moment of the shock arriving at $X=15 R_{E}$ (identified by a sudden increase in the IMF $B_{y}$ component) is set as Time $=0 \mathrm{~min}$ as a reference. It should be noted that at Time $=$ 2 min, a new open field line, as shown by the coarse blue line, appears, originating in the southern hemisphere, indicating the occurrence of high-latitude reconnection in the dawn quadrant of the southern hemisphere. 
Time $=-1 \mathrm{~min}$

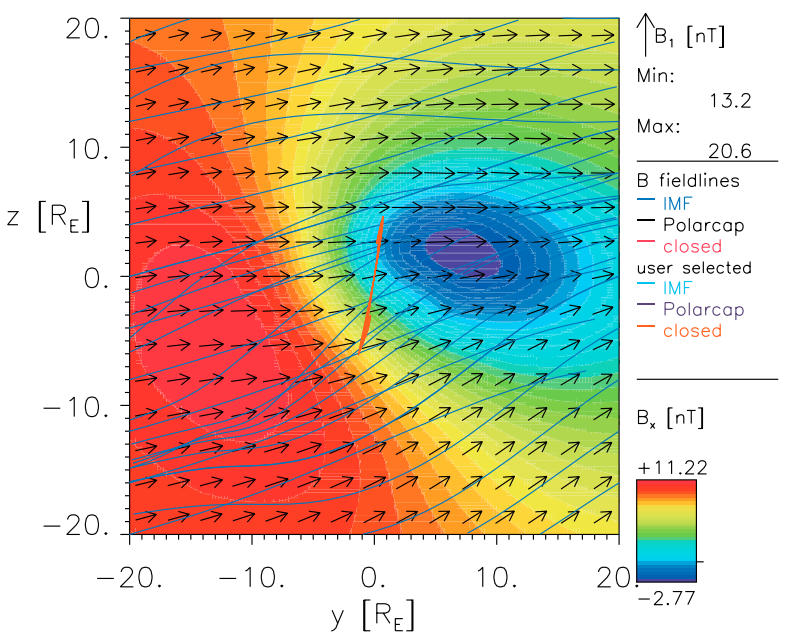

Time $=1 \mathrm{~min}$

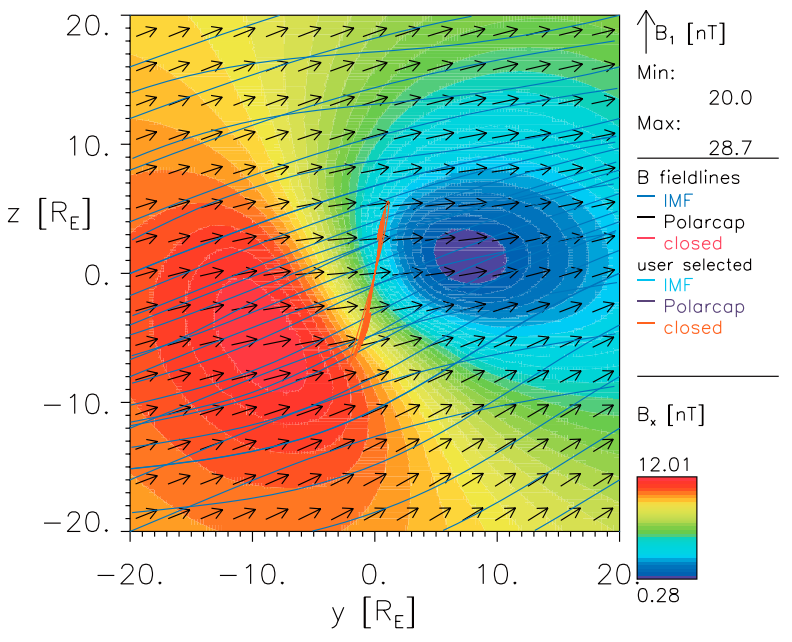

Time $=3 \mathrm{~min}$

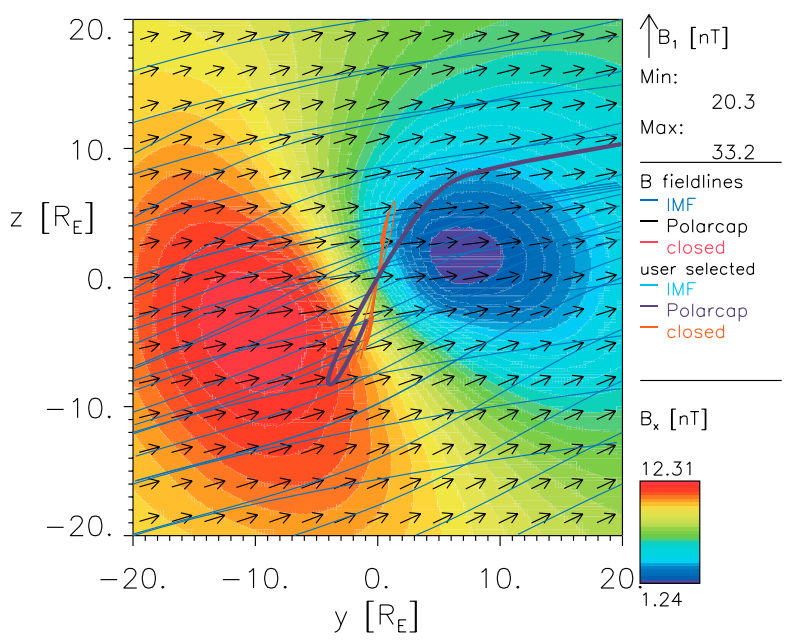

Time $=0 \mathrm{~min}$

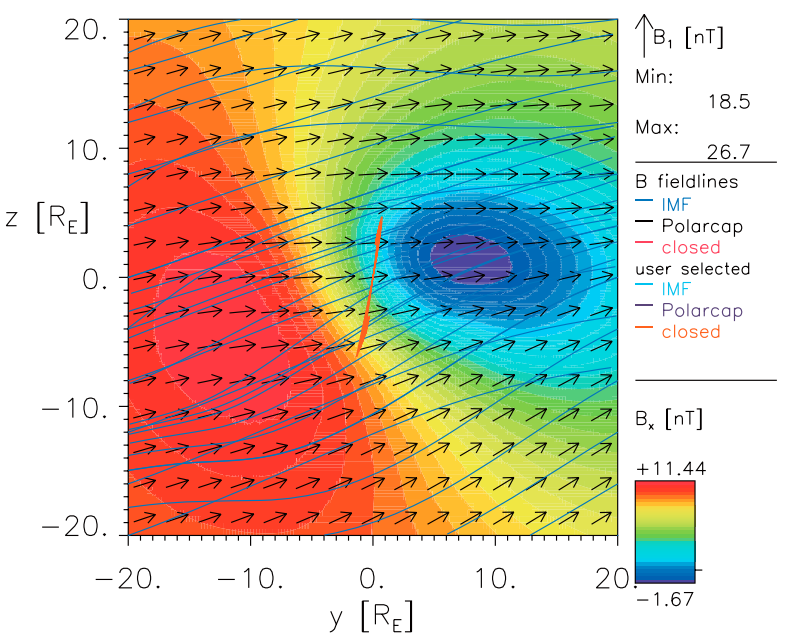

Time $=2 \mathrm{~min}$

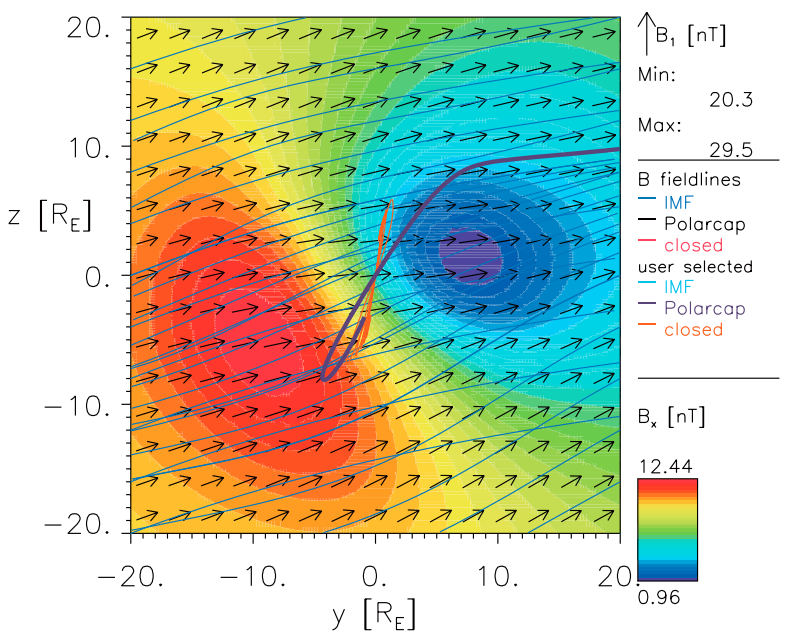

Time $=4 \mathrm{~min}$

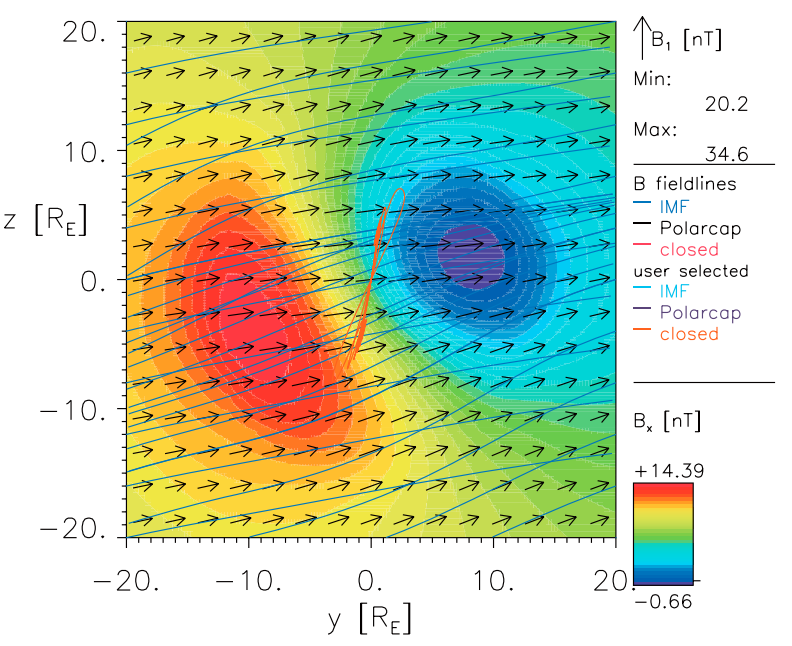

\section{Magnetic Field Topology of Real Event Simulation}

by the BATSRUS Model at CCMC (View at X=15 Re)

\section{Figure 8}




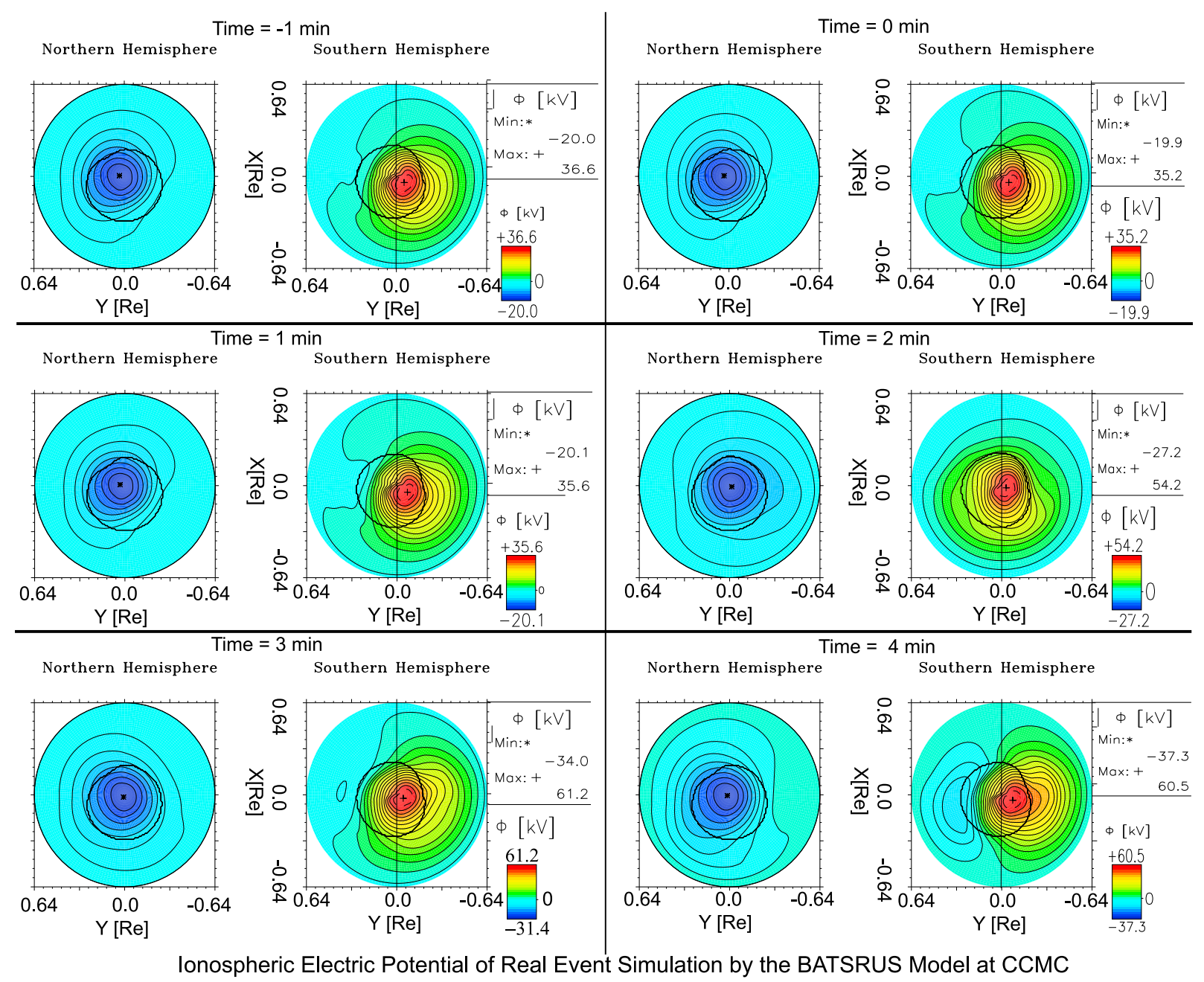

Figure 9. Real event simulation results of the BATSRUS model for the ionospheric electric potential in both hemispheres. Time $=0 \mathrm{~min}$ is set as the same moment as in Figure 8 . It should be noted that at Time $=2$ min, when the reconnection event is seen in Figure 8, the potential is suddenly increased in the southern ionosphere, and the high potential center moves from high to low latitude from Time $=2$ min to Time $=4 \mathrm{~min}$.

in the dawn quadrant of the southern hemisphere, Figure 5 shows nevertheless that there was no magnetic disturbance observed before time a at the LPM stations, which indicates that no significant reconnection occurred until the arrival of the shock. We therefore argue that we have presented clear observational evidence for the triggering of high-latitude reconnection by a shock under northward IMF $B_{z}$ condition.

\subsection{Significant Evidence for the Quasi-Instantaneous Transmission of the Polar Electric Field to the Dayside Dip Equator}

[28] Although Kikuchi et al. [1978] proposed that the polar electric field can instantaneously transmit to the dayside dip equator as the waveguide model, the validity of the model has subsequently been questioned [e.g., Chi et al., 2001, 2006] because of the lack of clear evidence for the simultaneous appearance of electric fields in both the polar region and the dayside dip equator. In this study, relying on observations with high time resolution and precise time stamp, we presented that the time difference of the precursor onset observed in the southern polar region and at the dayside dip equator is less than $10 \mathrm{~s}$ (Figure 5). Besides the precursor onset, Figures $5 \mathrm{a}$ and $5 \mathrm{~b}$ show that the PRI onset and the $N P T_{\mathrm{PRI}}$ observed at JIC were also coincident with two peaks of the variations observed at M79, M81, and M83. We thus argue that here we have presented some significant evidence for the validity of the waveguide model. Note that the timing of the simultaneous (uncertainty is less than $10 \mathrm{~s}$ ) appearance of the disturbance fields both in the polar region and at the dip equator presented in this study is extremely important for theoretically explaining the transmission of the polar electric field to the dayside dip equator.

\section{Summary and Conclusions}

[29] In this study, we examined a unique but significant SI event using extensive measurements of the geomagnetic field, together with spacecraft measurements and simulation results. What is unique is that the SI observed in the $H$ component at the dayside dip equator shows a clear precursor 

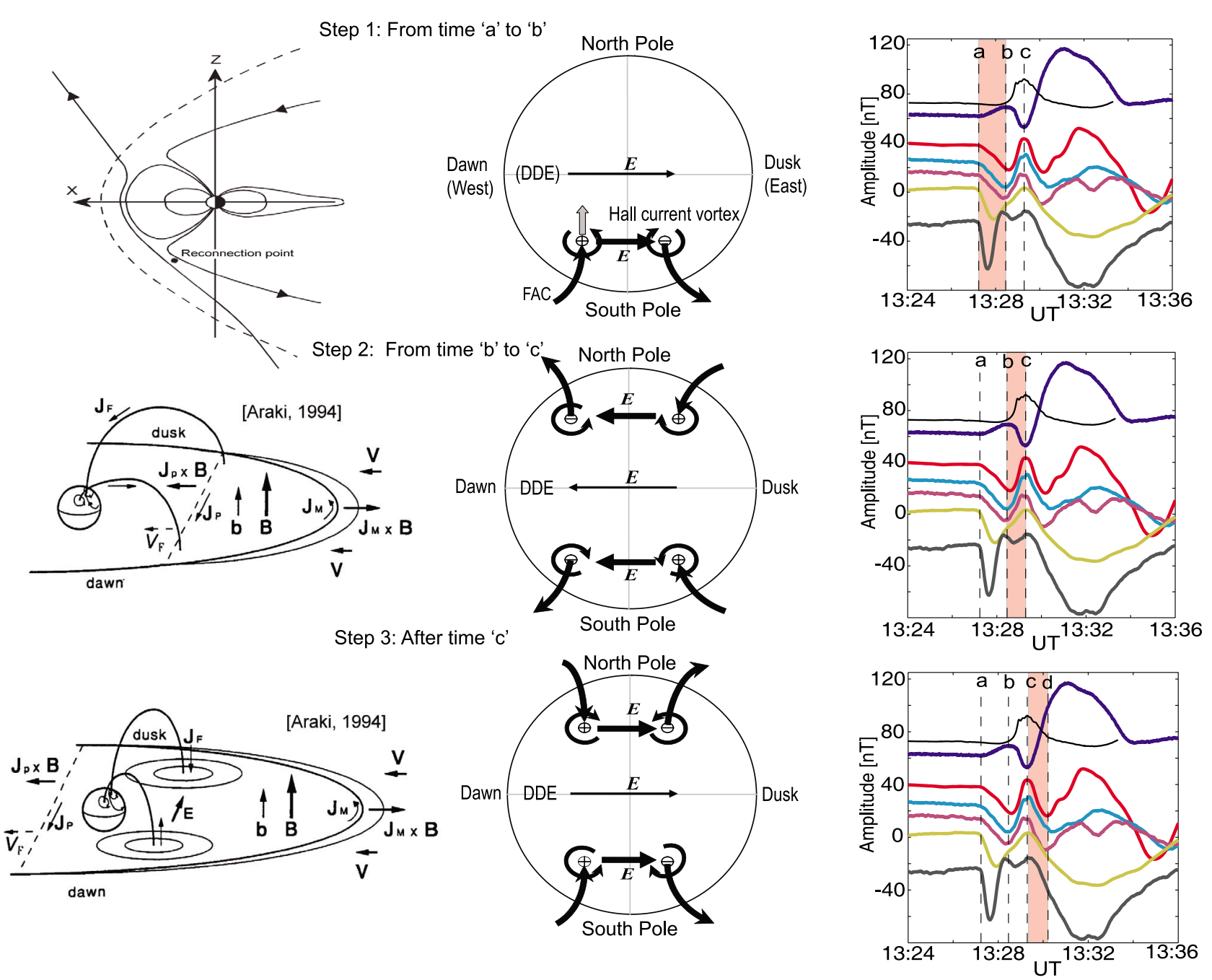

Figure 10. The processes schematically shown for generating the ground responses of the SI. The ionospheric Hall current vortexes are seen from above the Poles. From time a to time b, the observations consistent with a high-latitude reconnection occurred only in the dawn quadrant of the southern hemisphere. From time $\mathrm{b}$ to time $\mathrm{c}$ and after time $\mathrm{c}$, the ground responses consistent with the predictions of Araki's model for the $D P_{\mathrm{PI}}$ and $D P_{\mathrm{MI}}$ fields of SC/SI, respectively.

prior to the PRI onset. Taking advantage of global magnetic field observations, we confirmed that this SI precursor was simultaneously observed in the southern polar region and at the dayside dip equator but did not appear in the northern polar region at all, which implies that the SI precursor was caused by a nonconjugate process that occurred only, or first, in the southern hemisphere. Considering the IMF $B_{x}, B_{y}$, and $B_{z}$ components were all positive, we suggest that the nonconjugate process was high-latitude reconnection occurring in the dawn quadrant of the southern hemisphere. The real event simulation results by the BATSRUS model at CCMC are consistent with this suggestion. The resultant ionospheric effects of the simulation are well reflected in our observations. We therefore argue that high-latitude reconnection occurring only, or first, in the southern hemisphere is the most reasonable process for generating the precursor to the SI. Most importantly, this high-latitude reconnection effect was, for the first time, clearly identified at the dayside dip equator. In addition, we also stress that the high-latitude reconnection rate was enhanced by the arrival of the shock.
In future studies, we will focus on investigating how often such a precursor can be observed during the SC/SI period.

[30] Another important observational fact obtained from this study is the near-simultaneous appearance of the precursor onset both at the dip equator and in Antarctica. We stress that the uncertainty in the time difference of the precursor onset that can be identified in our observations between the polar region and the dip equator is less than $10 \mathrm{~s}$. We argue that these observational results present significant observational evidence for the waveguide model of Kikuchi et al. [1978], which suggests that the polar electric field can be quasi-instantaneously transmitted to the equator in the zeroth-order waveguide mode.

[31] Acknowledgments. This work was supported by the National Natural Science Foundation of China (NSFC grants 40974083, 40974103, and 40890164), the Open Research Program State Key Laboratory of Space Weather, CSSAR, CAS, and the National High Technology Research and Development Program of China (863 Program 2008AA121703). The authors thank UCLA Ground Magnetometer Data Center; World Data 
Center for Geomagnetism in Kyoto; Tromsø Geophysical Observatory University of Tromsø, Norway; Low Power Magnetometer (LPM) project of BAS; Sub-Auroral Magnetometer Network (SAMNET); CANadian Magnetic Observatory System (CANMOS); Magnetometer Array for Cusp and Cleft Studies (MACCS) of Canada; and Canadian Array for Realtime Investigations of Magnetic Activity (CARISMA) for supplying the ground magnetic field data. Simulation results have been provided by the Community Coordinated Modeling Center at Goddard Space Flight Center through their public Runs on Request system (http://ccmc.gsfc.nasa.gov). The CCMC is a multiagency partnership between NASA, AFMC, AFOSR, AFRL, AFWA, NOAA, NSF, and ONR. The BATSRUS Model was developed by Tamas Gombosi et al. at the Center for Space Environment Modeling, University of Michigan. The authors are grateful to K. Hosokawa for his help.

[32] Zuyin Pu thanks Athanasios Boudouridis and the other reviewers for their assistance in evaluating this paper.

\section{References}

Araki, T. (1994), A physical model of geomagnetic sudden commencement, in Solar Wind Sources of Magnetospheric Ultra-Low-Frequency Waves, Geophys. Monogr. Ser., vol. 81, edited by M. J. Engebretson, K. Takahashi, and M. Scholer, p. 183, AGU, Washington, D. C.

Araki, T., and H. Nagano (1988), Geomagnetic response to sudden expansions of the magnetosphere, J. Geophys. Res., 93(A5), 3983-3988.

Araki, T., et al. (1997), Anomalous sudden commencement on March 24 1991, J. Geophys. Res., 102(A7), 14,075-14,086.

Boudouridis, A., L. R. Lyons, E. Zesta, and J. M. Ruohoniemi (2007), Dayside reconnection enhancement resulting from a solar wind dynamic pressure increase, J. Geophys. Res., 112, A06201, doi:10.1029/ 2006JA012141.

Chi, P. J., et al. (2001), Propagation of the preliminary reverse impulse of sudden commencements to low latitudes, J. Geophys. Res., 106, $18,857-18,864$

Chi, P. J., D.-H. Lee, and C. T. Russell (2006), Tamao travel time of sudden impulses and its relationship to ionospheric convection vortices, J. Geophys. Res., 111, A08205, doi:10.1029/2005JA011578.

Cowley, S. W. H. (1983), Interpretation of observed relations between solar wind characteristics and effects at ionospheric altitudes, in High-Latitude Plasma Physics, edited by B. Hultqvist and T. Hagfors, pp. 225-249, Plenum, New York.

Fuselier, S. A., B. J. Anderson, and T. G. Onsager (1995), Particle signatures of magnetic topology at the magnetopause: AMPTE/CCE observations, J. Geophys. Res., 100(A7), 11,805-11,821.

Fuselier, S. A., B. J. Anderson, and T. G. Onsager (1997), Electron and ion signatures of field line topology at the low-shear magnetopause, J. Geophys. Res., 102(A3), 4847-4863.

Han, D.-S., and Q. Li (2008), Geomagnetic sudden commencement at the dawn-time dip equator, Earth Planets Space, 60, 607-612.

Han, D.-S., T. Iyemori, M. Nosé, H. McCreadie, Y. Gao, F. Yang, S. Yamashita, and P. Stauning (2004), A comparative analysis of low-latitude Pi2 pulsations observed by Ørsted and ground stations, J. Geophys. Res., 109, A10209, doi:10.1029/2004JA010576.

Han, D.-S., T. Araki, H.-G. Yang, Z.-T. Chen, T. Iyemori, and P. Stauning (2007), Comparative study of geomagnetic sudden commencement (SC) between Oersted and ground observations at different local times, J. Geophys. Res., 112, A05226, doi:10.1029/2006JA011953.
Kikuchi, T., and T. Araki (1979), Horizontal transmission of the polar electric field to the equator, J. Atmos. Terr. Phys., 41, 927-936.

Kikuchi, T., and T. Araki (2002), Comment on "Propagation of the preliminary reverse impulse of sudden commencements to low latitudes" by P. J. Chi et al., J. Geophys. Res., 107(A12), 1473, doi:10.1029/ 2001JA009220.

Kikuchi, T., T. Araki, H. Maeda, and K. Maekawa (1978), Transmission of polar electric fields to the equator, Nature, 273, 650-651.

Lavraud, B., M. F. Thomsen, M. G. G. T. Taylor, Y. L. Wang, T. D. Phan, S. J. Schwartz, R. C. Elphic, A. Fazakerley, H. Rème, and A. Balogh (2005), Characteristics of the magnetosheath electron boundary layer under northward interplanetary magnetic field: Implications for highlatitude reconnection, J. Geophys. Res., 110, A06209, doi:10.1029/ 2004JA010808.

Lavraud, B., M. F. Thomsen, B. Lefebvre, S. J. Schwartz, K. Seki, T. D. Phan, Y. L. Wang, A. Fazakerley, H. Rème, and A. Balogh (2006), Evidence for newly closed magnetosheath field lines at the dayside magnetopause under northward IMF, J. Geophys. Res., 111, A05211, doi:10.1029/2005JA011266.

Lockwood, M., and J. Moen (1999), Reconfiguration and closure of lobe flux by reconnection during northward IMF: Possible evidence for signatures in the cusp/cleft auroral emissions, Ann. Geophys., 17, 996-1011.

Russell, C. T., M. Ginskey, S. Petrinec, and G. Le (1992), The effect of solar wind dynamic pressure changes on low and mid-latitude magnetic records, Geophys. Res. Lett., 19, 1227-1230.

Russell, C. T., M. Ginskey, and S. M. Petrinec (1994a), Sudden impulses at low latitude stations: Steady state response for southward interplanetary magnetic field, J. Geophys. Res,. 99, 13,403-13,408.

Russell, C. T., M. Ginskey, and S. M. Petrinec (1994b), Sudden impulses at low-latitude stations: Steady state response for northward interplanetary magnetic field, J. Geophys. Res., 99, 253-261.

Takeuchi, T., T. Araki, H. Luehr, O. Rasmussen, J. Watermann, D. K. Milling, I. R. Mann, K. Yumoto, K. Shiokawa, and T. Nagai (2000), Geomagnetic negative sudden impulse due to a magnetic cloud observed on May 13, 1995, J. Geophys. Res., 105(A8), 18,835-18,846.

Twitty, C., T. D. Phan, G. Paschmann, B. Lavraud, H. Rème, and M. Dunlop (2004), Cluster survey of cusp reconnection and its IMF dependence, Geophys. Res. Lett., 31, L19808, doi:10.1029/2004GL020646.

Wang, C., J. B. Liu, Z. H. Huang, and J. D. Richardson (2007), Response of the magnetic field in the geosynchronous orbit to solar wind dynamic pressure pulses, J. Geophys. Res., 112, A12210, doi:10.1029/2007JA012664.

Watanabe, M., and G. J. Sofko (2009), Role of interchange reconnection in convection at small interplanetary magnetic field clock angles and in transpolar arc motion, J. Geophys. Res., 114, A01209, doi:10.1029/ 2008JA013426.

T. Araki, D.-S. Han, and H.-G. Yang, SOA Key Laboratory for Polar Science, Polar Research Institute of China, Shanghai, 200136, China. (handesheng@pric.gov.cn)

S. W. H. Cowley, Department of Physics and Astronomy, University of Leicester, LE1 7RH, Leicester, UK.

T. Iyemori, Data Analysis Center for Geomagnetism and Space Magnetism, Graduate School of Science, Kyoto University, 606-8502, Kyoto, Japan.

J. Liang, Department of Physics and Astronomy, University of Calgary, Calgary, AB, T2N 1N4, Canada. 